\title{
Challenges in reconciling observations and theory of the brightest high-energy flare ever of $3 \mathrm{C} 279$
}

\author{
Eugenio Bottacini*广 \\ Dipartimento di Fisica e Astronomia "G. Galilei", Universita di Padova, I-35131 Padova, Italy \\ W.W. Hansen Experimental Physics Laboratory \& Kavli Institute for Particle Astrophysics and \\ Cosmology, Stanford University, USA \\ E-mail: eugenio.bottacini@unipd.it
}

\section{Markus Böttcher}

Centre for Space Research, North-West University, Potchefstroom 2531, South Africa

\section{Elena Pian}

INAF, Istituto di Astrofisica Spaziale e Fisica Cosmica, via P. Gobetti 101, 40129 Bologna, Italy

Scuola Normale Superiore, Piazza dei Cavalieri 7, 56122 Pisa Italy

INFN, Sezione di Pisa, Largo Pontecorvo 3, 56127 Pisa, Italy

\section{Werner Collmar}

Max-Planck-Institut für extraterrestrische Physik, Giessenbach, 85748 Garching, Germany

\section{Dario Gasparrini}

Agenzia Spaziale Italiana (ASI) Space Science Data Center, I-00133, Roma, Italy Istituto Nazionale di Fisica Nucleare, sezione di Perugia, I-06123, Perugia, Italy

Recent high-energy missions have allowed keeping watch over blazars in flaring states, which provide deep insights into the engine powered by supermassive black holes. However, having a quasar caught in a very bright flaring state is not easy requiring long surveys. Therefore, the observation of such flaring events represents a goldmine for theoretical studies.

Such a flaring event was captured by the INTEGRAL mission in June 2015 while performing its (as of today) deepest extragalactic survey when it caught the prominent blazar 3C 279 in its brightest flare ever recorded at gamma-ray energies. The flare was simultaneously recorded by the Fermi gamma-ray mission, by the Swift mission, by the INTEGRAL mission and by observations ranging from UV, through optical to the near-IR bands. The derived snapshot of this broad spectral energy distribution of the flare has been modeled in the context of a one-zone radiation transfer leptonic and lepto-hadronic models constraining the single emission components. The derived parameters of both models challenge the physical conditions in the jet. However, very recently published very-high-energy (VHE) data at $\mathrm{TeV}$ energies are very close to our lepto-hadronic model.

35th International Cosmic Ray Conference - ICRC2017

10-20 July, 2017

Bexco, Busan, Korea

\footnotetext{
*Speaker.

${ }^{\dagger}$ NASA grant NNX13AO84G is acknowledged.
} 


\section{Introduction}

The flat spectrum radio quasar (FSRQ) 3C 279 has gained prominence due to its bright flaring state detected by the Energetic Gamma Ray Experiment Telescope (EGRET) on board the Compton Gamma-Ray Observatory (CGRO) mission [1]. This blazar, at redshift $z=0.5362$ [2], was the first (of currently only 5) FSRQs detected by ground-based Atmospheric Cherenkov Telescope facilities at very-high-energy (VHE: $E>100 \mathrm{GeV}$ ) gamma rays [3, 4]. This makes it an ideal target for multifrequency studies. Such extensive multifrequency campaigns [5] have allowed constraining important single emission components from the source [6].

Here we present the results from of the multifrequency campaign that pivots around the detection by the INTEGRAL mission of the brightest high-energy flare ever displayed by 3C 279 in June 2015 . We discuss the challenges faced by the modeling of the SED with a leptonic and a lepto-hadronic model shown in our recent work [7] and the implications of recently analyzed $\mathrm{TeV}$ data.

\section{Observations}

- INTEGRAL - In June 2015 INTEGRAL was performing its deepest extragalactic survey on the Come sky region. Due to the huge field of view of $29 \times 29 \mathrm{deg}^{2}$ the imager IBIS [8] detected the outburst of 3C 279 in only $50 \mathrm{ks}$ allowing us to compute a spectrum at energies above $15 \mathrm{keV}$. Data analysis is performed with the standard Off-line Scientific Analysis (OSA) software ${ }^{1}$ provided by the INTEGRAL Science Data Centre.

- Swift - During the INTEGRAL monitoring of 3C 279, the source was observed nearly simultaneously by Swift-XRT in photon counting mode on 2015-06-15 14:27 UTC (obs id: 00035019176), which allows for a detailed spectrum in the energy range $0.6-6.0 \mathrm{keV}$. In agreement with the very timely analysis by [9], we find the spectrum to be affected by pile-up due to the bright flare and the hard spectrum. After correcting for pile-up the spectral index is $\Gamma=-1.37$.

The same pointing by Swift led to observations with the UV-Optical Telescope (UVOT) with the $U$ filter.

- SMARTS - During the INTEGRAL monitoring the blazar 3C 279 was also targeted by the Moderate Aperture Research Telescope System (SMARTS) run by Yale University. Observations cover the optical to near-IR bands $B, V, R, J$, and $K$.

- FERMI - Also the Large Area Telescope (LAT) on board the Fermi gamma-ray mission captured the bright flare of the source. A prompt analysis of this detection [10] allowed for a precise spectrum and lightcurve of $3 \mathrm{C} 279$.

\section{Spectral Energy Distribution}

The spectral energy distribution (SED) of the sources displays the two broad non-thermal radiation components characteristic of blazars (see Figure 1). The low-energy component is due to synchrotron emission by relativistic electrons (possibly also positrons) in the jet, while the highenergy component can be either due to Compton scattering by the same relativistic electrons (lep-

\footnotetext{
${ }^{1} \mathrm{http} / / /$ www.isdc.unige.ch/integral/analysis\#Software
} 
tonic processes) or due to proton synchrotron radiation and synchrotron emission from secondaries produced in photo-pion interactions (hadronic processes).

To model this boradband SED, we adopt the two approaches: a leptonic and a hadronic model. We use their time-independent homogeneous one-zone jet radiation transfer [11], building upon an earlier work [12].

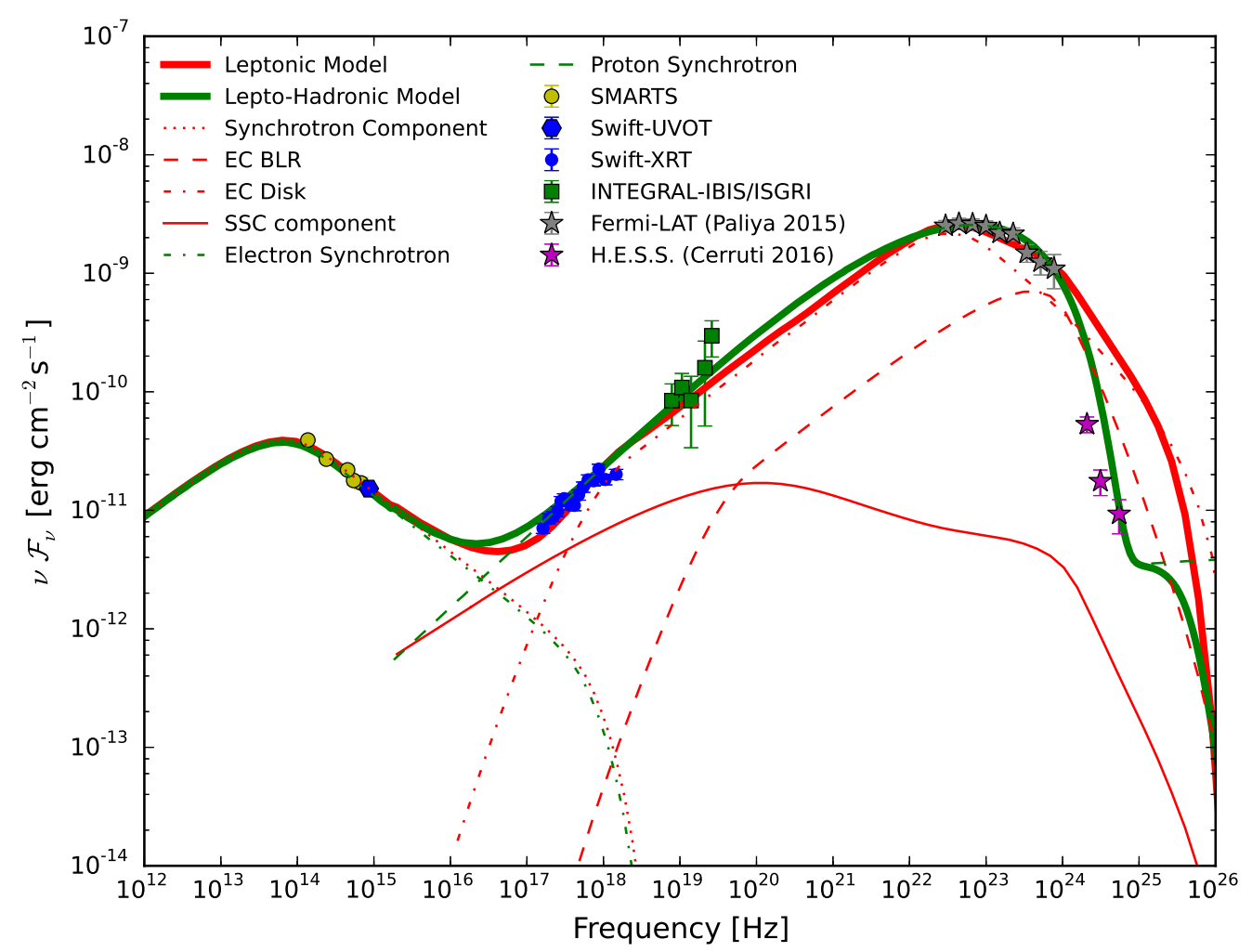

Figure 1: Quasi-simultaneous SED of 3C 279, along with the leptonic (red solid) model and the leptohadronic (green solid) model, as described in the text. The different emission components for both models as shown too (see legend). The data from the H.E.S.S. collaboration were not included in the modeling. However, they line up surprisingly well with the lepto-hadronic model.

\section{Discussion and Conclusions}

The SED can be equally well modeled by a leptonic and by a lepto-hadronic model (see Figure 1). However, both models challenge the physical conditions in the jet.

The leptonic model indicates that the emission region is dominated by kinetic energy by a factor of $\sim 10$ compared to the energy carried in the magnetic field. This may be difficult to realize in a jet in which the magnetic field is the primary source of jet power because one would expect any mechanism converting magnetic-field to particle kinetic energy to cease once equipartition is reached. In the leptonic scenario the emission region is inferred to be well within the broad line reagion (BLR), thus VHE photons would not escape this region unattenuated [13]. Therefore, the 
leptonic model predicts that no VHE photons should be detected in this flare, if not originating at a different location.

On the other hand, our lepto-hadronic SED model allows us to choose parameters close to equipartition between the magnetic field and the relativistic proton population. An important issue for this model may be the extreme jet power, of the order of the Eddington luminosity of the central black hole in 3C 279.

Because 3C 279 is one of currently only 5 FSRQs detected at VHE, the source is subject to a ToO program by H.E.S.S. triggered on the basis of publicly available blazar observations. Here we adapt the data of the 3C 279-flare presented in a very recent analysis by the H.E.S.S. collaboration [14]. The data are shown in Figure 1. These data line up surprisingly well with our lepto-hadronic model.

\section{References}

[1] Hartman, R. C., Bertsch, D. L., Fichtel, C. E., et al. 1992, ApJL, 385, L1

[2] Marziani, P., Sulentic, J. W., Dultzin-Hacyan, D., Calvani, M., \& Moles, M. 1996, ApJS, 104, 37

[3] Albert, J., Aliu, E., Anderhub, H., et al., 2008, Science, 320, 1752

[4] Mirzoyan, R. 2015, The Astronomer's Telegram, 7080

[5] Collmar, W., Böttcher, M., Krichbaum, T. P., et al. 2010, A\&A, 522, A66

[6] Pian, E., Urry, C. M., Maraschi, L., et al. 1999, ApJ, 521, 112

[7] Bottacini, E., Böttcher, M., Pian, E., \& Collmar, W. 2016, ApJ, 832, 17

[8] Ubertini, P., Lebrun, F., Di Cocco, G., et al. 2003, A\&A, 411, L131

[9] Pittori, C., Verrecchia, F., Puccetti, et al. 2015a, The Astronomer's Telegram, 7639

[10] Paliya, V. S. 2015, ApJL, 808, L48

[11] Böttcher, M., Reimer, A., Sweeney, K., \& Prakash, A. 2013, ApJ, 768, 54

[12] Böttcher, M., \& Chiang, J. 2002, ApJ, 581, 127

[13] Böttcher, M., \& Els, P. 2016, ApJ, 821, 102

[14] Cerruti, M., Böttcher, M., Chakraborty, N., et al. 2016, arXiv:1610.05523 